\title{
PREVENTION OF PULMONARY VASCULAR AND MYOCARDIAL REMODELING BY THE COMBINED TYROSINE AND SERINE-/THREONINE KINASE INHIBITOR, SORAFENIB, IN PULMONARY HYPERTENSION AND RIGHT HEART FAILURE
}

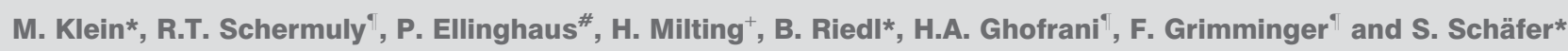

François Brenot Award, sponsored by AstraZeneca

*Cardiology Research, Bayer Schering Pharma, Wuppertal, Germany

\#Target discovery, Bayer Schering Pharma, Wuppertal, Germany

University of Giessen Lung Center, Giessen, Germany

+Heart- \& Diabetes Center NRW, Bad Oeynhausen, Germany

WINNING ABSTRACT: Inhibition of tyrosine kinases can reverse pulmonary hypertension but little is known about the role of serine-/threonine kinases in vascular and myocardial remodeling. We investigated the effects of sorafenib, an inhibitor of the tyrosine kinases VEGFR, PDGFR and c-kit as well as the serine-/threonine kinase Raf-1, in pulmonary hypertension and right ventricular (RV) pressure overload.

In monocrotaline treated rats, sorafenib $\left(10 \mathrm{mg} \cdot \mathrm{kg}^{-1} \cdot \mathrm{d}^{-1}\right.$ p.o.) reduced pulmonary arterial pressure, pulmonary artery muscularization and RV hypertrophy, and improved systemic hemodynamics (table 1). Sorafenib prevented phosphorylation of Raf-1 and suppressed activation of downstream signaling pathways (Erk 1/2).

After pulmonary banding, sorafenib, but not the PDGFR/c-KIT/ABL-inhibitor imatinib reduced RV mass and RV filling pressure significantly. Congruent with these results, sorafenib only prevented ERK phosphorylation and vasopressin induced hypertrophy of the cardiomyocyte cell line $\mathrm{H} 9 \mathrm{c} 2$ dose dependently (IC50=300 nM).

Combined inhibition of tyrosine and serine-/threonine kinases by sorafenib prevents vascular and cardiac remodeling in pulmonary hypertension, which is partly mediated via inhibition of the Raf kinase pathway.

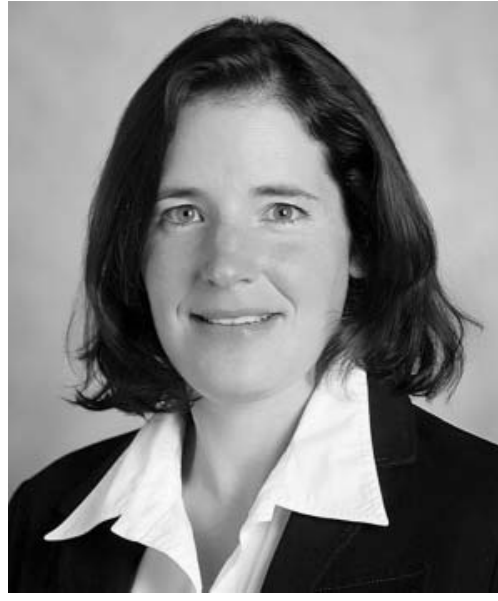

Martina Klein

Cardiology Research, Bayer Schering Pharma, Wuppertal, Germany

\section{MY JOB AND THE UNIT IN WHICH I WORK}

I work in the Pre-clinical Cardiology Research Dept at Bayer Schering Pharma (Wuppertal, Germany). Within this group, my scientific interest focuses on the mechanisms of pulmonary vascular and myocardial remodelling and the validation and discovery of new drugs for pulmonary hypertension and heart failure.

STATEMENT OF INTEREST: None declared
MY WINNING ABSTRACT AS A PART OF MY RESEARCH

Pulmonary arterial hypertension is a life-threatening disease characterised by deviations in signal transduction, and proliferation and migration of pulmonary endothelium and smooth muscle cells, resembling cancer pathophysiology. Current therapeutic approaches are based on vasodilatory mechanisms, which include local and systemic administration of prostacyclin and its analogues, endothelin receptor antagonists [1] and phosphodiesterase- 5 inhibitors. Although therapeutic targeting of these pathways has proven efficacy in clinical trials, a sustained regression of the disease cannot usually be achieved. Hence, in addition to these vasodilatory mechanisms, vascular remodelling must be approached via

TABLE 1 Control and monocrotaline (MCT) treated rats

\begin{tabular}{lccc} 
& Control & MCT+vehicle & MCT+sorafenib \\
\hline RVP $\mathbf{~ m m H g}$ & $27.0 \pm 0.5^{*}$ & $82.9 \pm 6.0$ & $35.0 \pm 1.5^{*}$ \\
RVEDP $\mathbf{~ m m g}$ & $2.0 \pm 0.2^{*}$ & $7.7 \pm 1.3$ & $2.6 \pm 0.2^{*}$ \\
$\mathbf{R V} /(\mathbf{L V}+\mathbf{S})$ & $0.24 \pm 0.01^{*}$ & $0.51 \pm 0.02$ & $0.26 \pm 0.01^{*}$ \\
$\mathbf{C O} \mathbf{~ m L} \cdot \mathbf{m i n}^{-1}$ & $116.1 \pm 5.1^{*}$ & $70.5 \pm 6.7$ & $117.4 \pm 5.3^{*}$ \\
$\mathbf{P a}, \mathbf{O}_{\mathbf{2}} \mathbf{~ m m H g}$ & $204.8 \pm 18.5^{*}$ & $107.7 \pm 13.8$ & $200.0 \pm 10.4^{*}$ \\
\hline
\end{tabular}

Data are presented as mean \pm SEM. RVP: right ventricular pressure; RVEDP right ventricular end-diastolic pressure; $R V /(L V+S)$ : ratio of right ventricle to left ventricle plus sputum; $\mathrm{CO}$ : cardiac output; $\mathrm{Pa}_{\mathrm{a}} \mathrm{O}_{2}$ : arterial oxygen tension. In each group, $n=12-14 .{ }^{*}: p<0.05$ versus vehicle. 
more causal mechanisms. Therefore, we investigated the novel multi-kinase inhibitor sorafenib, which inhibits the tyrosine kinases vascular endothelial growth factor receptor, plateletderived growth factor receptor and c-kit, as well as the serine/ threonine kinase Raf-1, in pulmonary hypertension and right ventricular pressure overload.

\section{MY RESEARCH AS PART OF MY WORKING GROUP/ RESEARCH TEAM}

Cardiology is one of the four core research focuses of Bayer Schering Pharma, the others are oncology, female healthcare and diagnostic imaging. Within cardiology research, pulmonary hypertension is one of the key research areas. In this area, Bayer Schering Pharma has a rich pipeline of compounds in pre-clinical and clinical development, including iloprost, which is marketed as Ventavis.
THE IMPACT OF MY WORK ON CLINICAL OR RESEARCH PRACTICE

New treatments are needed for pulmonary arterial hypertension. Current therapeutic approaches mainly provide symptomatic relief, as well as some improvement of prognosis, but there is no cure. Median survival is $4-5$ yrs from the time of diagnosis. Our studies have helped to better understand the cellular mechanisms involved in the pathogenesis of pulmonary arterial hypertension, a process that to date remains unclear. Ultimately, this knowledge allows us to develop new therapeutic strategies for this complex and lethal disease with focus on anti-remodelling processes.

\section{REFERENCES}

1 Schermuly RT, Pullamsetti SS, Kwapiszewska G, et al. Phosphodiesterase 1 upregulation in pulmonary arterial hypertension. Circulation 2007; 115: 2331-2339. 